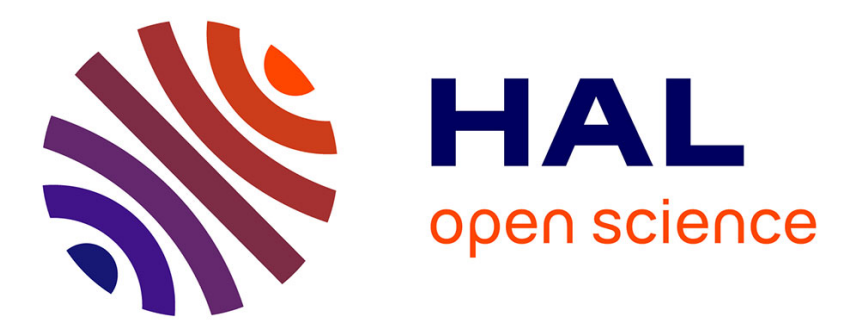

\title{
Utilisation de supports visuels et de l'induction linguistique pour répondre aux besoins d'apprenants en anglais de spécialité
}

\author{
Corinne Landure
}

\section{- To cite this version: \\ Corinne Landure. Utilisation de supports visuels et de l'induction linguistique pour répondre aux besoins d'apprenants en anglais de spécialité. Recherche et Pratiques Pédagogiques en Langues de Spécialité: Cahiers de l'APLIUT, 2012, Image et enseignement/apprentissage des langues : le cas du secteur LANSAD, 31 (1), pp.28-43. 10.4000/apliut.2246 . halshs-01246737}

\section{HAL Id: halshs-01246737 \\ https://shs.hal.science/halshs-01246737}

Submitted on 19 Dec 2015

HAL is a multi-disciplinary open access archive for the deposit and dissemination of scientific research documents, whether they are published or not. The documents may come from teaching and research institutions in France or abroad, or from public or private research centers.
L'archive ouverte pluridisciplinaire HAL, est destinée au dépôt et à la diffusion de documents scientifiques de niveau recherche, publiés ou non, émanant des établissements d'enseignement et de recherche français ou étrangers, des laboratoires publics ou privés. 
Corinne Landure, «Utilisation de supports visuels et de l'induction linguistique pour répondre aux besoins d'apprenants en anglais de spécialité », Recherche et pratiques pédagogiques en langues de spécialité [En ligne], Vol. XXXI N 1 | 2012, mis en ligne le 15 février 2012, consulté le 28 mars 2015. URL : http://apliut.revues.org/2246; DOI : 10.4000/apliut.2246

Les apprenants en entreprise sont souvent décrits par leurs formateurs comme étant plutôt motivés et impliqués dans l'acquisition d'une langue seconde (L2) alors que, d'après leurs enseignants, les étudiants LANSAD (LANgues pour Spécialistes d'Autres Disciplines) sembleraient moyennement s'investir et montreraient un intérêt modéré dans l'apprentissage d'une L2. Ce décalage est en partie attribué à la maturité des apprenants en entreprise et s'expliquerait également par des nécessités ou contraintes à caractère professionnel auxquelles serait soumis ce public évoluant dans un contexte et un environnement toujours plus exigeants (Dubar 2004). Seraient-ce pour autant les seules raisons? En entreprise, par exemple, on accorde beaucoup d'importance à l'analyse des besoins des futurs apprenants car celle-ci permet de mettre en place un programme d'apprentissage personnalisé afin de cibler davantage leurs objectifs. Comme les attentes de ces apprenants sont précises et souvent pressantes, il en résulte des pratiques d'apprentissage spécifiques. Si, dans le milieu professionnel, une concertation entre apprenants et formateurs pour la détermination des besoins semble faire son chemin et s'inscrire dans une pratique régulière, qu'en est-il dans le contexte universitaire? De quelles façons l'enseignant ajuste-t-il ses pratiques d'enseignement en fonction des besoins et attentes exprimés par les étudiants et que peut-il en résulter au niveau des pratiques d'apprentissage d'apprenants d'une L2 ?

Répondre à ces questions est l'objectif de cette étude qui s'articule autour de deux axes. Tout d'abord, un état des lieux portant sur la formation en anglais dispensée dans six entreprises lorraines, et des cours d'anglais donnés dans un Institut Universitaire de Technologie (IUT) du nord-est de la France. Les besoins, les attentes et les pratiques d'apprentissage dans ces deux contextes spécifiques y sont particulièrement visés. Les données recueillies proviennent de réponses à des entretiens (annexe 1) réalisés en entreprise auprès de salariés de niveau Bac+2 bénéficiant d'une formation continue dispensée au sein de l'entreprise par des intervenants extérieurs dans le cadre de la formation professionnelle tout au long de la vie. Ces informations sont ensuite comparées aux réponses des étudiants en $1^{\mathrm{e}}$ et $2^{\mathrm{e}}$ années de DUT Génie Industriel et Maintenance (GIM). Le deuxième axe de cette étude concerne une expérience sur le terrain impliquant ces mêmes étudiants dans la mise en œuvre d'un projet initié par eux et qui tient compte de leurs besoins et attentes. Une analyse qualitative fondée sur l'observation de leurs réactions et l'enregistrement de leurs commentaires renseigne sur leur implication dans ce projet ; les résultats quantitatifs nous informent sur l'efficacité linguistique de l'utilisation et de la combinaison d'outils et de ressources (supports visuels et corpus) au service de l'apprentissage de l'anglais de spécialité.

\section{1. État des lieux des besoins et attentes d'apprenants en anglais de spécialité}

L'un des objectifs de cette étude est de comparer les besoins, les attentes et les pratiques d'apprentissage des apprenants dans le contexte professionnel et à I'IUT. Pour ce faire, une trentaine d'apprenants en entreprise, titulaires d'un Bac+2 et suivant actuellement une formation en anglais de spécialité, ont été contactés ; lors de ces entretiens, des questions concernant leurs besoins, leurs attentes et le déroulement de leurs cours ont été abordées. Les mêmes questions ont ensuite été posées à 32 étudiants en $1^{\mathrm{e}}$ et $2^{\mathrm{e}}$ années de DUT GIM afin de pouvoir procéder à une comparaison des résultats.

Il ressort de cette étude que les besoins principaux des apprenants en entreprise sont de trois ordres; ils se situent au niveau de l'acquisition d'une compétence rédactionnelle (rédaction de messages électroniques ou de courriers formels), de l'aptitude à expliquer des consignes (au téléphone ou lors de déplacements à l'étranger) et de la capacité à comprendre un document professionnel. Les étudiants, quant à eux, demandent une remise à niveau en grammaire et souhaitent acquérir le vocabulaire technique lié à leur domaine de spécialité dans le but de pouvoir s'exprimer simplement, de savoir faire des phrases aussi bien à l'écrit qu'à l'oral. Cette conception de la langue peut sembler naïve voire réductrice au premier abord ; elle peut s'expliquer par la non 
prise de conscience de l'utilisation de la langue de spécialité en milieu professionnel, notamment en raison de leur absence d'expérience dans ce domaine; effectivement, à ce stade de leur cursus, aucun étudiant n'a encore effectué de stage en entreprise. Le rôle de l'enseignant est alors essentiel de les amener à prendre en compte d'autres perspectives liées à l'apprentissage de la langue de spécialité allant au-delà de leurs préconisations initiales.

Les apprenants en entreprise attendent des cours ciblés et personnalisés tenant compte de leur fonction et spécialité. Ils privilégient un apprentissage axé sur la résolution de tâches concrètes ayant un rapport direct avec leur travail en entreprise. Leur objectif est de devenir autonomes en anglais dans leurs fonctions respectives. Les étudiants de I'IUT, quant à eux, apprécient que les cours se centrent sur le domaine technique; ils préconisent néanmoins que ceux-ci soient plus axés sur leur spécialité et qu'ils visent davantage leur future fonction, en y incluant des tâches plus concrètes par exemple. Leur objectif est d'être opérationnels en entreprise dès l'embauche.

Quant au déroulement de la formation et aux pratiques d'apprentissage en entreprise, les cours sont dispensés en petits groupes ne dépassant généralement pas trois participants ; de ce fait, ceux-ci trouvent que l'apprentissage se révèle plus efficace. Les tâches sont spécifiques à l'entreprise et à la fonction, là aussi pour une plus grande efficacité. Il n'est pas rare que le formateur se déplace dans les ateliers pour mieux se rendre compte des besoins des techniciens ou qu'il travaille avec eux sur des documents authentiques propres à l'entreprise. Les apprenants préfèrent cibler une ou deux compétences langagières à la fois, dans un souci d'efficacité pour les besoins propres à la fonction. À l'IUT où cette étude s'est déroulée, les cours d'anglais sont répartis en travaux dirigés (TD) composés de 20 étudiants maximum et en travaux pratiques (TP) comprenant 10 étudiants au plus. Sur les cours visant leur domaine de spécialité (de manière assez large) viennent se greffer des cours plus traditionnels avec de la grammaire et des exercices d'application. L'utilisation de documents pédagogiques semble être privilégiée, mais apparemment ceux-ci seraient peu appréciés par les étudiants qui les trouvent ennuyeux et peu pertinents, parce que quelquefois trop éloignés de leurs centres d'intérêts. Toutes les compétences langagières sont abordées dans les cours et les étudiants trouvent cette mixité de compétences à acquérir difficile et contraignante.

En entreprise, généralement, le programme de formation est validé par le directeur des ressources humaines (DRH) ou le responsable de formation en concertation avec les apprenants, suivant les besoins de ces derniers. Pourtant, certains directeurs des ressources humaines ont une conception divergente de ce que devrait intégrer le programme pour une formation en langues. C'est ce que révèlent les réponses de six responsables de formation ou ressources humaines des entreprises où travaillent les apprenants que nous avons interrogés. Les DRH préconisent des cours dans le domaine de spécialité, mais avec des exercices de grammaire. Ils préfèrent l'utilisation de supports pédagogiques et du livre de grammaire plutôt que l'exploitation de documents authentiques. Les apprenants devraient, selon eux, travailler toutes les compétences langagières, même s'ils n'en utilisent qu'une ou deux dans leur fonction et ils s'attendent à ce que les apprenants soient autonomes rapidement. On remarque donc un certain décalage entre la conception de la formation d'une L2 vue par les DRH et celle d'apprenants directement confrontés aux besoins du terrain. Malgré ces divergences de vues, les DRH laissent à leurs salariés l'initiative de gérer leur apprentissage selon leurs propres besoins dans la mesure où les compétences acquises répondent aux critères de la fonction et au degré d'efficacité attendu par l'entreprise.

Pour rappel, les principaux besoins exprimés par les étudiants de l'IUT sont les suivants : remise à niveau en grammaire, acquisition du vocabulaire technique, capacité à faire des phrases à l'écrit et à l'oral et des cours plus concrets dans leur domaine de spécialité. L'étude présentée dans cet article a pour deuxième objectif de répondre à leurs besoins et attentes exprimés lors du questionnaire, en leur donnant les moyens de devenir les acteurs de leur propre apprentissage. Nous nous appliquerons ainsi, dans un deuxième temps, à évaluer leur implication dans ce processus et à suivre les étapes de leur cheminement dans leur quête d'un apprentissage conçu pour eux et surtout par eux, sous la houlette discrète ou l'impulsion avisée de l'enseignante.

\section{Méthodologie}

Cette étude comprend, d'une part, l'observation des pratiques des apprenants dans leur démarche d'apprentissage semi-autonome visant la mise en œuvre d'un projet suite à l'analyse de leurs besoins et, d'autre part, l'analyse des résultats linguistiques provenant de la consultation d'un corpus générique pour l'autocorrection de leurs productions. 
Cette expérience, s'échelonnant sur six séances de deux heures, a été conduite dans un IUT avec la participation de 14 étudiants en première année et 18 en deuxième année d'études en Génie Industriel et Maintenance. Malgré leurs cinq à huit années d'anglais scolaire, leur niveau est assez modeste, comme l'indiquent les résultats à un test informel du TOEIC (moyenne de 395 points sur 990) effectué quelques semaines auparavant, et se situe approximativement au niveau A2 / B1 du CECR (Cadre Européen Commun de Référence). Dans le cadre de cette étude, chaque étudiant dispose d'un ordinateur et d'un accès à Internet pour la consultation de diverses ressources en ligne.

Deux instruments de recherche ont été utilisés pour les besoins de cette expérience. Le premier consiste en l'observation des réactions des apprenants relevées ou enregistrées par l'enseignante (annexe 2), et le second concerne les résultats linguistiques de leurs travaux de traduction qui combinent I'utilisation de ressources traditionnelles et la consultation de corpus en ligne. L'analyse des résultats linguistiques renseigne quant à la nature des consultations et des modifications effectuées et permet d'évaluer dans quelle mesure l'apprentissage linguistique par l'exploitation de corpus (ALEC) ou, comme l'appelle Johns (1991) le data-driven learning $(D D L)$, contribue à améliorer la production des apprenants et leur autonomisation dans le processus d'apprentissage de la langue.

\section{Déroulement de l'expérience}

Les résultats qualitatifs sont fondés sur I'observation des réactions et le relevé des commentaires des participants lors des différentes phases. Notre attention s'est plus particulièrement portée sur les trois premières séances (tableau 1). La première séance implique les deux classes lors de la phase de réflexion, la seconde porte sur la phase liée à l'organisation au sein de chaque classe et, enfin, la troisième séance concerne le travail en binôme au sein de chaque classe pour l'utilisation des ressources se centrant sur les travaux de rédaction et de traduction.

Tableau 1 : Phases et séances

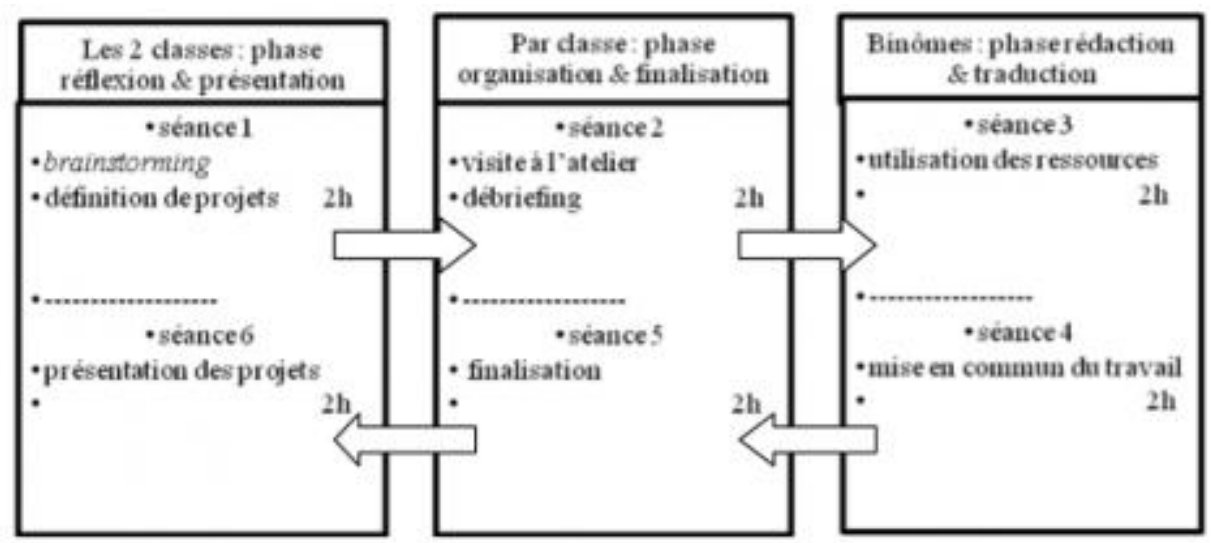

\subsection{Phase réflexive : cheminement vers la créativité}

Afin de discuter des résultats provenant des réponses données quant à leurs besoins et attentes dans l'apprentissage d'une L2, une réunion est organisée avec les deux classes qui se transforme rapidement en un brainstorming; l'objectif de cette rencontre, se déroulant en français, est de produire des idées, de les combiner, de les associer afin de mieux répondre à leurs besoins et à leurs attentes. Après avoir présenté les résultats du questionnaire aux étudiants, l'enseignante, qui ne souhaite pas orienter leurs idées et choix, n'intervient que sur la forme du débat mais pas sur le fond; c'est-à-dire qu'elle ne prend pas directement part à la discussion, elle entre en scène uniquement pour préciser des termes, reformuler des idées, dégager des pistes et relancer la discussion. Après une heure de discussion, engagée timidement dans les premiers temps, il s'ensuit une libération de paroles, principalement chez les étudiants de deuxième année, avec une volonté marquée de s'impliquer. On observe que les termes revenant régulièrement durant cette séance sont : « vocabulaire », « utile», « concret», « authentique », 《créatif », « technique » et « grammaire ». De ce débat résulte un accord entériné par les deux classes sur la définition et les modalités de réalisation d'un projet commun: la création de catalogues et d'affiches. Il est convenu que les apprenants en première année s'occuperaient de la création d'un catalogue et 
d'affiches répertoriant les outils de la boîte à outils de l'atelier et que les étudiants en deuxième année procéderaient de façon identique avec les machines se trouvant dans l'atelier de I'IUT. Grâce à cette discussion, ils ont abouti à une forme d'apprentissage par des tâches (Ellis $2000 ; 2003$ ). Comment en sont-ils arrivés là ? La majorité des étudiants pense qu'il est plus facile de retenir le vocabulaire technique en l'associant avec une photo, une image ou un croquis que d'apprendre des listes de vocabulaire spécifique ou de ne rencontrer qu'un certain nombre de mots dans les textes. Pour autant, certains font remarquer que nommer un outil ou une machine n'est pas suffisant, et qu'il est nécessaire de pouvoir en décrire l'aspect, la fonction. D'autres, se projetant dans leurs futures fonctions de techniciens de maintenance, avancent l'idée qu'une réflexion sur d'éventuels problèmes, comme l'anticipation de pannes possibles, permettrait d'aborder des préoccupations réelles et répondrait ainsi à l'un des aspects concrets de leurs futures professions. Sont également évoqués des problèmes habituels ou ponctuels rencontrés dans leur atelier; ils mentionnent notamment leurs désaccords sur l'utilisation de telle machine ou leurs hésitations quant au fonctionnement de telle autre. De cette réflexion est né le projet de créer un catalogue bilingue français / anglais répertoriant outils et machines ainsi que des affiches qui seraient exposés à I'atelier à la vue de tous. Ces supports visuels auraient pour particularité de décrire l'outil en plus de le nommer et, pour les machines, d'indiquer de façon simple et concise leur utilisation, leur fonctionnement, l'équipement de sécurité adjoint, les pannes et usures éventuelles (annexes 3 et 4). Cette manière simpliste de reproduire la langue de spécialité pourrait servir de tremplin pour aborder le discours à visée professionnelle, évidemment plus complexe dans sa représentation.

\subsection{Phase organisationnelle : inventaire des ressources matérielles}

Pour l'inventaire des ressources matérielles, une visite à l'atelier est organisée pour chaque classe afin de répertorier, classer, photographier les différents outils et machines. Six classes d'outils ou petites pièces de la boîte à outils et sept machines de l'atelier ont été retenues. Il s'ensuit un débriefing au sein de chaque classe, dont l'objectif est de composer les groupes afin de répartir les tâches, de répertorier les ressources à disposition et de fixer le cahier des charges pour la réalisation du projet.

Les étudiants vont ensuite travailler sur une classe d'outil ou une machine en particulier, sachant que chacun dispose d'un ordinateur, de logiciels et de divers périphériques informatiques en plus d'un accès à Internet. Leurs tâches, à ce stade, intéressent autant la recherche documentaire technique que la conception visuelle pour qu'ensuite une mise en commun des travaux aboutisse à la réalisation des supports définitifs. Les étudiants doivent également s'accorder sur une présentation commune pour une harmonisation visuelle de l'ensemble. On remarque beaucoup d'enthousiasme et de nombreux échanges durant cette phase. Godinet (2007) avance que pour qu'il y ait collaboration, deux préalables doivent être réunis, le premier étant d'avoir des objectifs et des besoins proches, le second de partager des valeurs semblables.

\subsection{Phase rédactionnelle : utilisation des ressources linguistiques}

Quant aux ressources linguistiques, elles se composent tout d'abord de leurs propres connaissances, que les étudiants ont souvent tendance à sous-estimer au profit d'autres ressources comme les traducteurs en ligne, qu'ils pourront malgré tout consulter. Ils ont à leur disposition également des glossaires, des dictionnaires bilingues ainsi que l'accès à un corpus générique en ligne, le BNC(British National Corpus). L'utilisation d'un corpus générique comme ressource complémentaire aux ressources traditionnelles n'est pas une nouveauté pour ces étudiants ; ils avaient été initiés quelques semaines auparavant à l'exploitation de corpus pour l'autocorrection des travaux de traduction (Landure \& Boulton 2010). Les corpus en ligne présentent de nombreux avantages, notamment leur accessibilité immédiate et gratuite. Ils répondent également aux besoins individuels et spécifiques des apprenants et leur utilisation se fait directement, sans intermédiaire et sans contraintes de temps et d'espace. Par ailleurs, ils ne nécessitent que peu d'investissement tant matériel que formatif. L'apprentissage linguistique par l'exploitation de corpus (ALEC) offre aux apprenants la possibilité de diriger et de cibler leur propre apprentissage, de construire de manière inductive leurs connaissances. De plus, il permet de développer I'autonomisation des apprenants comme l'indiquent les nombreuses études recensées par Boulton (2010). 


\section{Exploitation des ressources et induction linguistique pour accomplir la tâche}

Pour tous travaux de production écrite, le premier réflexe des étudiants est de se précipiter sur les traducteurs en ligne, bien qu'ils sachent pertinemment que les résultats obtenus ainsi sont loin d'être fiables. À défaut de pouvoir leur interdire complètement de les utiliser, il leur est conseillé, dans un premier temps, de mobiliser leurs propres connaissances et ensuite, dans un souci de développer leur sens critique, de consulter au moins trois traducteurs en ligne afin de comparer et d'évaluer les résultats obtenus. Leurs connaissances, ainsi que les traducteurs, sont la trame ou le premier jet de leur production qu'ils doivent ensuite améliorer en consultant d'autres ressources, comme les dictionnaires et les glossaires, afin de préciser et d'affiner des termes lexicaux par exemple. Enfin, ils sont invités à exploiter un corpus généraliste en ligne, le British National Corpus $(B N C)$, pour auto-corriger leur traduction.

L'objectif est de les amener à construire méthodiquement leur réflexion linguistique par l'exploitation de diverses ressources à leur disposition pour un résultat final qui, à défaut d'être exempt d'erreurs, sera le fruit d'un raisonnement, d'un cheminement tant individuel que collaboratif. Travaillant en binômes au sein de la classe, ils sont évidemment encouragés à communiquer leurs stratégies. On peut ainsi remarquer un échange d'informations et d'astuces aussi bien que l'expression d'avis divergents portant sur la manière de procéder ou de raisonner. On peut évoquer ici une collaboration contradictoire où l'accent est essentiellement mis sur la manière d'arriver au résultat plutôt que sur le résultat final lui-même (Baudrit 2007).

Les deux exemples de phrases ci-dessous restituent la production de deux étudiants. Pour une meilleure lisibilité des résultats, il leur avait été suggéré de souligner en vert les items consultés dans les dictionnaires ou glossaires (ici en italique) et en rouge les items consultés dans le corpus (ici soulignés). La dernière phrase représente le résultat final, fruit des réflexions des étudiants suite à leurs diverses consultations.

\section{Exemples}

1. PHRASE ORIGINALE : Dans un premier temps, on place la piece brute sur la table et on la serre dans l'étau à l'aide des mors.

SYSTRAN : Initially, one places the blank on the table and one tightens it in the vice using the bits.

GOOGLE : Firstly, place the blank on the table and the greenhouse in the vise with the jaws.

REVERSO : At first, we place the raw room on the table and we squeeze it in the vice by means of bits.

PHRASE FINALE : Initially, we place the blank on the table and we tighten it in the vice using the jaws.

2. PHRASE ORGINALE: Dans un deuxième temps, on déplace la fraise avec les commandes du pupitre pour tailler la pièce selon le dessin technique.

SYSTRAN : In the second time, one moves the strawberry with the orders of the desk to cut the part according to the technical drawing.

GOOGLE: In a second step, the cutter moves with the control booth to cut the piece by drawing technique.

REVERSO : Secondly, we move the strawberry with the commands of the writing desk to cut the room according to the technical drawing.

PHRASE FINALE : Secondly, one moves the mill with the control panel to cut the part according to the technical design.

Comme l'illustre l'exemple ci-dessus, en cas de divergence de résultats provenant des traducteurs, et plutôt que d'opter pour un choix arbitraire ou intuitif, l'étudiant choisit une autre alternative en optant pour la consultation du corpus qui, par l'exploration des données, lui permet de trouver par ses propres moyens, sans l'aide de l'enseignante, une réponse appropriée. Dans d'autres cas, la consultation lui sert à confirmer un résultat préalablement trouvé dans une autre ressource ou, tout simplement, lui permet de conforter son intuition. Il est intéressant de noter que le taux de consultation des items dans le corpus s'élève à $24 \%$, alors que la recherche dans le dictionnaire représente $16 \%$ et que le résultat lié à I'utilisation des traducteurs atteint $60 \%$. Les consultations du corpus ont été analysées en fonction des modifications effectuées; en d'autres termes, on a comptabilisé les modifications négatives qui ont abouti à un résultat erroné, les modifications positives qui ont conduit à une traduction appropriée et les absences de modification qui ont peutêtre permis de conforter un résultat préalablement trouvé avec les autres ressources. Comme 
I'indique le tableau 2 ci-dessous, on obtient $10 \%$ de modifications négatives, $41 \%$ de modifications positives et $49 \%$ de modifications zéro.

Tableau 2 : Consultation de corpus et modifications

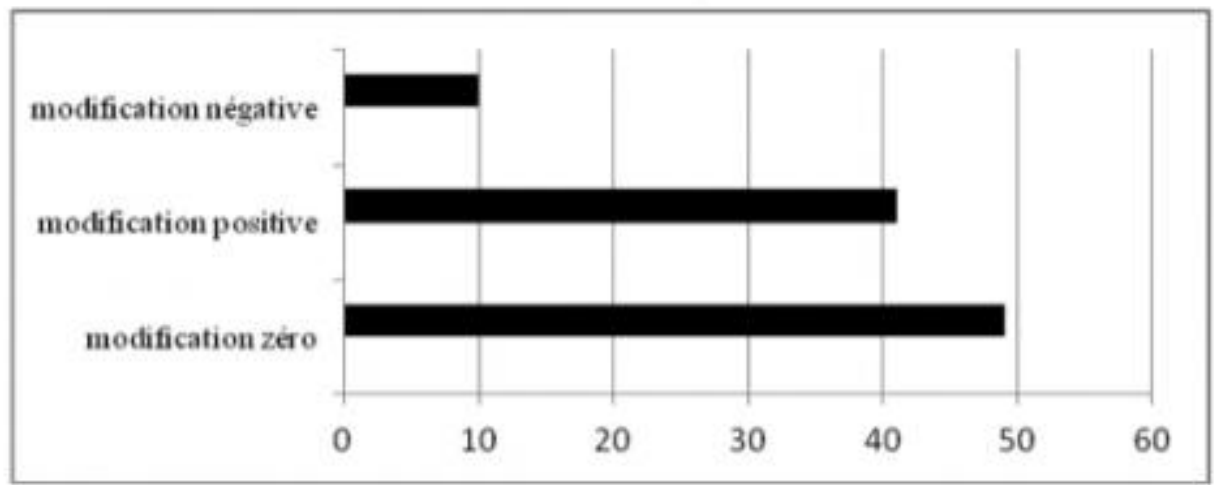

Les $41 \%$ de modifications positives, provenant de l'autocorrection de leurs productions par les étudiants (tableau 3), portent principalement sur les collocations (47\%) qui sont probablement moins repérables dans les traducteurs ou dictionnaires. Nous trouvons ensuite les prépositions (22 \%) qui peuvent être plus faciles à localiser grâce au co-texte et contexte qu'offre le corpus et enfin suivent les expressions idiomatiques et la syntaxe à hauteur de $11 \%$ et $8 \%$ respectivement.

\section{Tableau 3 : Autocorrection}

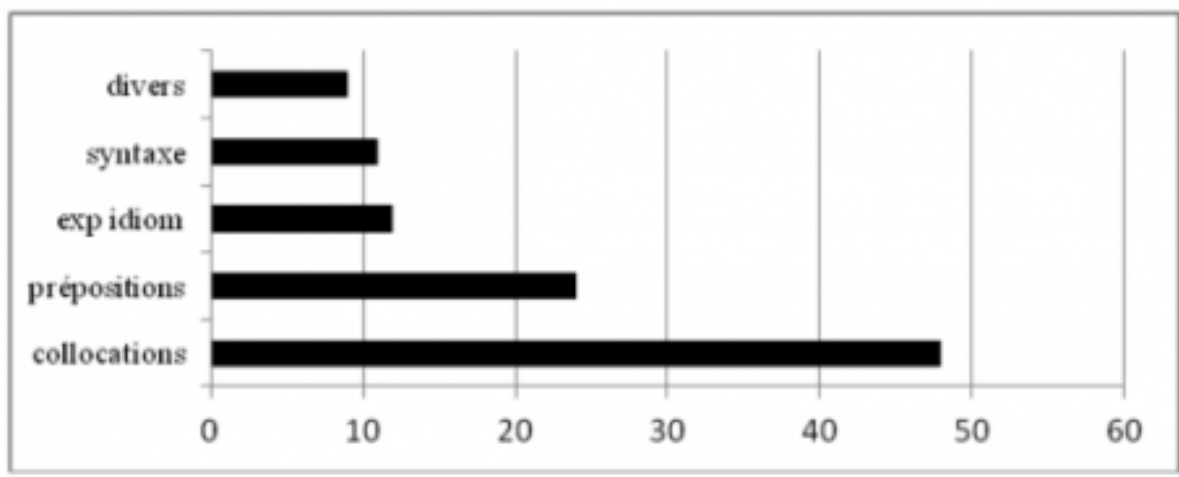

\section{Conclusion}

Cette étude montre que la prise en compte des besoins d'apprenants L2 peut favoriser la motivation d'étudiants LANSAD en les impliquant dans une démarche raisonnée d'apprentissage semi-autonome ou autonome en sachant que l'autonomie est l'une des compétences les plus appréciées en entreprise. L'expression des besoins par les apprenants, quels que soient l'environnement et le contexte de formation, semble indispensable à tout apprentissage productif. Pour autant, il est important de noter que les besoins ne sont pas figés et qu'ils peuvent évoluer en fonction de différents facteurs (Brown 2009). L'idéal serait de prendre en considération et de combiner les besoins subjectifs exprimés par les apprenants et les besoins objectifs émis par d'autres intervenants comme les décideurs institutionnels, les enseignants ou les directeurs des ressources humaines. Les enseignants / chercheurs, en tant que spécialistes à l'écoute des apprenants et des acteurs du monde professionnel, ont également un rôle essentiel à jouer pour faire évoluer les représentations et mettre en place des dispositifs plus adaptés sur le court et le plus long terme. Dans notre cas, on s'aperçoit que les étudiants de I'IUT ont conçu collaborativement un projet mêlant images et textes, intégrant des tâches qui trouvent leur origine 
dans l'expression de leurs besoins subjectifs et qui correspondent à leurs attentes du moment. Ils ont fait preuve à la fois de créativité et de raisonnement - créativité dans la conception initiale du projet jusque dans sa réalisation finale, et raisonnement dans l'adoption d'une démarche inductive lors de la consultation de corpus pour l'autocorrection de leur production écrite.

La pratique d'activités collaboratives fondées sur des tâches intégrant les TIC relance non seulement la motivation des étudiants mais favorise également l'autonomisation et la dynamique de groupe, donnant une impulsion nouvelle à l'apprentissage d'une L2. Si cette approche interactive centrée sur l'apprenant a pu les conduire à devenir plus autonomes dans leur apprentissage en s'attachant à répondre à l'expression de leurs besoins, il n'est pas sûr en revanche qu'ils aient réalisé à quel point les connaissances ainsi acquises peuvent s'appliquer dans le domaine professionnel puisqu'ils n'ont encore eu que très peu de contacts avec le monde du travail. Il serait intéressant de faire se rencontrer des étudiants, des salariés et des apprenants en milieu professionnel pour aborder les questions de l'apprentissage d'une L2 de spécialité afin d'aller plus loin dans la réflexion sur la définition des réels besoins en milieu professionnel. La participation des professeurs de langue lors de ces échanges apparaît essentiel, tant pour prendre conscience des réalités du terrain que pour préparer la voie vers des pratiques d'apprentissage de la langue de spécialité plus appropriées. 


\section{Bibliographie}

Baudrit, A. 2007. L'apprentissage collaboratif: plus qu'une méthode collective ?Bruxelles: De Boeck.

Boulton, A. 2010. «Learning outcomes from corpus consultation ». InMoreno, M. Jaén, F. Serrano Valverde \& M. Calzada Pérez (eds.). Exploring New Paths in Language Pedagogy: Lexis and Corpus-Based Language Teaching. Londres : Equinox, 129-144. Supplément électronique disponible à : http://arche.univ-nancy2.fr/course/view.php ?id $=967$.

Brown, J. 2009. «Foreign and second language needs analysis ». In Long, M. \& C. Daughty (eds.). The Handbook of Language Teaching. Chichester: Wiley-Blackwell, Chapter 16. DOI : $10.1002 / 9781444315783 . c h 16$

BYU-BNC : British National Corpus. http://corpus.byu.edu/bnc/x.asp. Consulté en mai 2011.

Coquard, A. \& M. Le Nir. 2010. Le devenir des diplômés de DUT: septième enquête nationale. Direction Générale de I'Enseignement Supérieur en collaboration avec les IUT. http://www.iutfr.net/publications/enquete-ip.html. Consulté en novembre 2011.

Conseil de I'Europe. 2001. Cadre européen commun de référence pour les langues : apprendre, enseigner, évaluer. Paris : Didier.

Dubar, C. 2004. La formation professionnelle continue. Paris : La Découverte.

Ellis, R. 2000. «Task-based research and language pedagogy ». Language Teaching Research, vol $14, n^{\circ} 3: 193-220$.

Ellis, R. 2003. Task-based language learning and teaching. Oxford : Oxford University Press.

Godinet, H. 2007. «Scénario pour apprendre en collaborant à distance : contraintes et complexité ». In Le campus numérique FORSE : analyses et témoignages. Rouen : Publication des Universités de Rouen et du Havre, 113-129.

Johns, T. 1991. «Should you be persuaded : two samples of data-driven learning ». In Johns, T. \& P. King (eds.). Classroom Concordancing : English Language Research Journal, $n^{\circ} 4$. University of Birmingham, 1-16.

Landure, C. \& A. Boulton. 2010. «Corpus et autocorrection pour l'apprentissage des langues ». $A S p$, $n^{\circ} 57$ : 11-30. DOI : $10.4000 / a s p .931$

Test of English for International Communication. http://www.toeic.eu/.Consulté en mai 2011. 
Société :

Service :

Nom :

Prénom :

Profession :

Langue choisie :

Analyse des besoins professionnels pour 2008

(Entourer les réponses)

Utilisation de la langue

au téléphone accueil/convivialité réunion voyage d'affaires

lecture de documents professionnels rédaction de documents professionnels

autre (à préciser)

Emploi de la langue

tous les jours $\quad 1$ fois par semaine $\quad 1$ fois par mois jamais

Objectif de la formation : quelles compétences souhaitez-vous développer ou améliorer?

compréhension orale expression orale compréhension écrite

expression écrite grammaire vocabulaire spécifique autre :

À court terme :

A moyen terme :

A long terme :.

Fréquence de la formation

$1 \times 1$ h30/semaine $\quad 1 \times 1$ h30 tous les 15 jours $1 \times 4 h /$ mois autre (à préciser)

Outils pédagogiques : quels supports pédagogiques aimeriez-vous utiliser ?

livre de cours cahier d'exercices K7 ou CD

vos propres supports (documents internes) autres (à préciser)

Caractéristiques de la formation : recherchez-vous une formation :

type scolaire (leçons et exercices) plutôt beaucoup de grammaire

plutôt beaucoup d'oral (conversationnel) plutôt beaucoup d'écrit (rédactionnel)

axée sur les connaissances culturelles plutôt technique autre : 


\section{Annexe 2 - Grilles d'observation $\mathbf{1}^{\mathrm{e}}$ année GIM}

\begin{tabular}{|c|c|c|c|c|c|c|c|}
\hline Nom & $\begin{array}{l}\text { Prise de } \\
\text { parole }\end{array}$ & mots clés & Interruption & $\begin{array}{l}\text { Marque de } \\
\text { l'aceord }\end{array}$ & $\begin{array}{l}\text { Marque de } \\
\text { desaccord }\end{array}$ & $\begin{array}{c}\text { Idées } \\
\text { notvelles }\end{array}$ & $\begin{array}{l}\text { Capacite } \\
\text { de } \\
\text { persuasion }\end{array}$ \\
\hline \multicolumn{8}{|l|}{$\mathrm{AU}$} \\
\hline \multicolumn{8}{|l|}{$\mathrm{TI}$} \\
\hline \multicolumn{8}{|l|}{ KAM } \\
\hline \multicolumn{8}{|l|}{$\mathrm{JU}$} \\
\hline \multicolumn{8}{|l|}{$\mathrm{KA}$} \\
\hline \multicolumn{8}{|l|}{ MI } \\
\hline \multicolumn{8}{|l|}{ IS } \\
\hline \multicolumn{8}{|l|}{$\mathrm{VI}$} \\
\hline \multicolumn{8}{|l|}{ LA } \\
\hline \multicolumn{8}{|l|}{ JE } \\
\hline \multicolumn{8}{|l|}{$\mathrm{KE}$} \\
\hline \multicolumn{8}{|l|}{$\mathrm{CH}$} \\
\hline \multicolumn{8}{|l|}{ LO } \\
\hline MA & & & & & & & \\
\hline
\end{tabular}

REMARQUES

\begin{tabular}{|c|c|c|c|c|c|c|c|}
\hline Nom & $\begin{array}{l}\text { Participa }{ }^{\circ} \\
\text { Inventaire }\end{array}$ & $\begin{array}{c}\text { Degré } \\
\text { d'implica }\end{array}$ & $\begin{array}{c}\text { Preuve } \\
\text { d'initiative }\end{array}$ & $\begin{array}{l}\text { Utilisa }^{\circ} \\
\text { multimédia }\end{array}$ & $\begin{array}{l}\text { Degré de } \\
\text { collabora }^{\circ}\end{array}$ & $\begin{array}{l}\text { Réparti }^{\circ} \\
\text { des täches }\end{array}$ & $\begin{array}{c}\text { Degré } \\
\text { d'autonomie }\end{array}$ \\
\hline \multicolumn{8}{|l|}{$\mathrm{AU}$} \\
\hline \multicolumn{8}{|l|}{$\mathrm{TI}$} \\
\hline \multicolumn{8}{|c|}{ KAM } \\
\hline \multicolumn{8}{|l|}{ JU } \\
\hline \multicolumn{8}{|l|}{ KA } \\
\hline \multicolumn{8}{|l|}{ MI } \\
\hline \multicolumn{8}{|l|}{ IS } \\
\hline \multicolumn{8}{|l|}{$\mathrm{VI}$} \\
\hline \multicolumn{8}{|l|}{ LA } \\
\hline \multicolumn{8}{|l|}{ JE } \\
\hline \multicolumn{8}{|l|}{$\mathrm{KE}$} \\
\hline \multicolumn{8}{|l|}{$\mathrm{CH}$} \\
\hline \multicolumn{8}{|l|}{ LO } \\
\hline $\mathrm{MA}$ & & & & & & & \\
\hline
\end{tabular}

GRILLE PHASE 3 / BINOME/ UTILISATION DES RESSOURCES

\begin{tabular}{|l|l|l|l|l|l|l|l|}
\hline Nom & \multicolumn{3}{|c|}{ Utilisation du } & \multicolumn{3}{c|}{ Collaboration } \\
\hline & dico & traducteuf & glossaire & corpus & active & moyenne & inexistante \\
\hline AU & & & & & & & \\
\hline TI & & & & & & & \\
\hline KAM & & & & & & & \\
\hline JU & & & & & & \\
\hline KA & & & & & & \\
\hline MI & & & & & & \\
\hline IS & & & & & & \\
\hline VI & & & & & & \\
\hline LA & & & & & & \\
\hline JE & & & & & & \\
\hline KE & & & & & & & \\
\hline CH & & & & & & & \\
\hline LO & & & & & & & \\
\hline MA & & & & & & \\
\hline
\end{tabular}




\section{Annexe 3 - Exemple de machine}

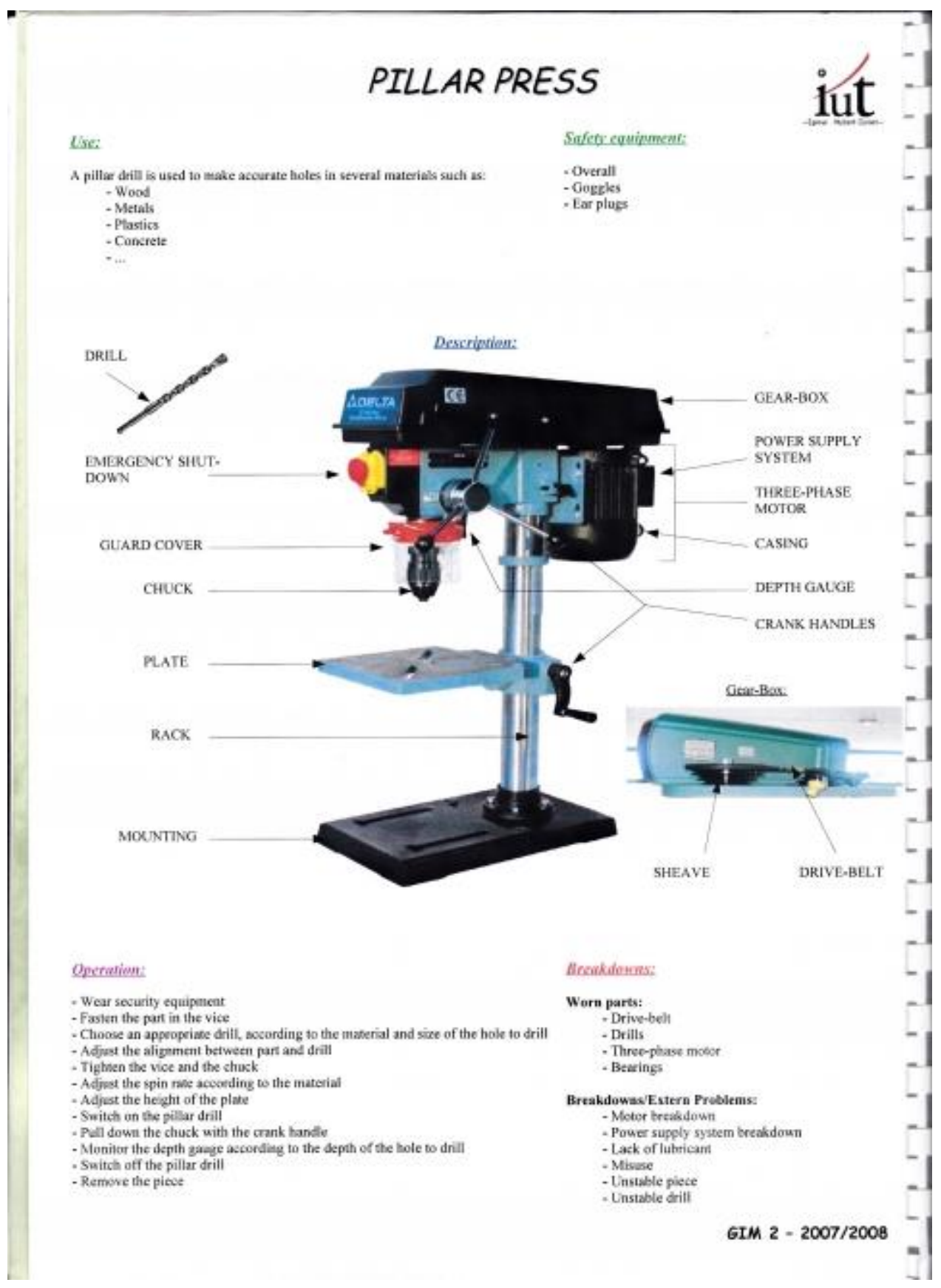




\section{Annexe 4 - Exemple d'outils}

[

Nancy-Université

Gim 1 (2007-2008)

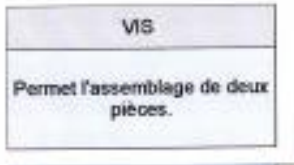

Screw

Mows the assembly of two parts.

Vis a tete hexagonal

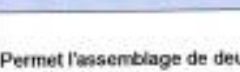

Permet l'assemblage de deux pueces.

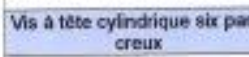

Permet rassemblage de deux pieces.

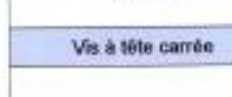

Permet rassemblage de deux pitones.

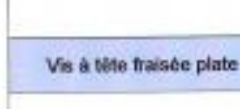

Permet Fassemblage de deux

piéces.

Vs a wete bombse fendu

Permet rassemblage de deux

Permet rassemblas
pitoes.
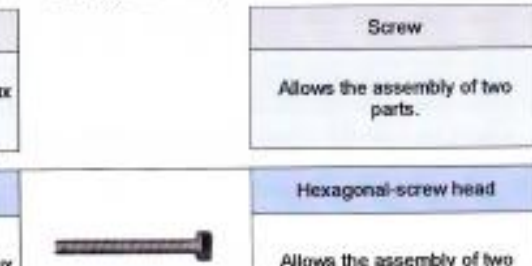

Hexagonal-screw head

Allowa the assembly of two parts

Alows the assembly of two parts

Allows the assembly of two parts.

Miled caps purt screw

Nlows the assembly of two parts.

Truss head screw spit

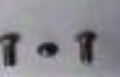

Allows the assembly of two parts.

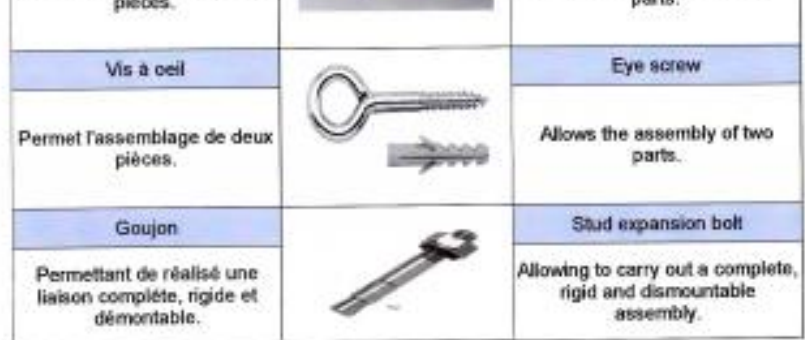

\section{Notes}

$\underline{\mathbf{1}}$ Les résultats d'une enquête nationale (Coquard \& Le Ny 2010) sur l'insertion professionnelle conduite par la Direction Générale de I'Enseignement Supérieur en collaboration avec les IUT (2010) indiquent que près de $20 \%$ des étudiants ayant obtenu leur DUT en Génie Industriel et Maintenance en 2007 ont bénéficié d'une embauche immédiate et durable. Cette étude souligne également que $70 \%$ des autres étudiants en GIM avaient, quant à eux, choisi de poursuivre leurs études. 